Article

\title{
Factors Associated with Attitudes toward Aging among Taiwanese Middle-Aged and Older Adults: Based on Population-Representative National Data
}

\author{
Shu-Hsin Lee ${ }^{1,2,3}$, Chih-Jung Yeh ${ }^{4}$, Cheng-Yu Yang ${ }^{5}$, Ching-Yi Wang ${ }^{5,6, *}$ and Meng-Chih Lee ${ }^{3,7,8,9, * \mathbb{D}}$ \\ 1 Department of Nursing, Chung Shan Medical University, Taichung 402306, Taiwan; shl@csmu.edu.tw \\ 2 Department of Nursing, Chung Shan Medical University Hospital, Taichung 402306, Taiwan \\ 3 Institute of Medicine, Chung Shan Medical University, Taichung 402306, Taiwan \\ 4 Department of Public Health, Chung Shan Medical University, Taichung 402306, Taiwan; \\ alexyeh@csmu.edu.tw \\ 5 Department of Physical Therapy, Chung Shan Medical University, Taichung 402306, Taiwan; \\ clack088688@gmail.com \\ 6 Physical Therapy Room, Chung Shan Medical University Hospital, Taichung 402306, Taiwan \\ 7 Department of Family Medicine, Taichung Hospital, Ministry of Health and Welfare, Taichung 403301, Taiwan \\ 8 Institute of Population Health Sciences, National Health Research Institutes, Miaoli 350401, Taiwan \\ 9 College of Management, Chaoyang University of Technology, Taichung 413310, Taiwan \\ * Correspondence: cywang@csmu.edu.tw (C.-Y.W.); mcl@csmu.edu.tw (M.-C.L.)
}

check for

updates

Citation: Lee, S.-H.; Yeh, C.-J.; Yang, C.-Y.; Wang, C.-Y.; Lee, M.-C. Factors Associated with Attitudes toward Aging among Taiwanese Middle-Aged and Older Adults: Based on Population-Representative National Data. Int. J. Environ. Res. Public Health 2022, 19, 2654. https:// doi.org/10.3390/ijerph19052654

Academic Editor: Paul B. Tchounwou

Received: 23 January 2022

Accepted: 24 February 2022

Published: 24 February 2022

Publisher's Note: MDPI stays neutral with regard to jurisdictional claims in published maps and institutional affiliations.

Copyright: (c) 2022 by the authors. Licensee MDPI, Basel, Switzerland. This article is an open access article distributed under the terms and conditions of the Creative Commons Attribution (CC BY) license (https:// creativecommons.org/licenses/by/ $4.0 /)$.

\begin{abstract}
In middle-aged and older adults, attitude toward aging (ATA) exerts significant influences on their current and future health. For health promotion to be successful, participants' ATA requires health care providers' attention. Knowing the factors associated with ATA can facilitate future studies to investigate effective interventions. The aim of this study was to identify the factors associated with ATA in middle-aged and older adults. This cross-sectional study analyzed data of a nationally representative sample of adults aged 58 years and older collected in a population-based longitudinal study: the Taiwan Longitudinal Study on Aging (TLSA). To identify the factors associated with ATA, we investigated demographic factors (age, gender, education, marital and cohabitation status, and financial satisfaction status) and health-related factors (number of co-morbidities, depression, physical function dependency) with bivariate analysis and multiple regression analysis. To identify the activities beneficial to ATA over and above demographic and health-related factors, various activities (exercise, volunteer service, and leisure activities) were each examined individually by multiple regression analysis. The factors detrimental to ATA were advanced age, a higher number of co-morbidities, living alone, depression, and dependence on physical function. Those beneficial to ATA were higher education, financial satisfaction, physical exercise, volunteer service, and six leisure activities.
\end{abstract}

Keywords: attitude toward aging; leisure activities; physical exercise; volunteer service

\section{Introduction}

Attitude toward aging (ATA) refers to one's beliefs about aging and older people in general and it is gradually built up through a process of assimilation from one's surrounding culture and internalized self-relevant attitudes and stereotypes across one's life span [1]. One's ATA significantly influences one's health. In the literature, ATA is often measured by questionnaires such as Attitudes toward own Aging subscale of the Philadelphia Geriatric Center Morale Scale (PGCMS) [2], Attitudes to Aging Questionnaire (AAQ) [3], and Expectations Regarding Aging (ERA-12) [4]. Studies consistently show that negative ATA, assessed by the above mentioned questionnaires, is associated with poorer health, less control over one's lifestyle behaviors, poorer functioning performance, and lower life satisfaction [5-8]. More importantly, this relationship is found not only in cross-sectional 
studies but also in longitudinal investigations, and it exists in both middle-aged and older adults [9-12]. People with negative ATA are less likely to engage in an active lifestyle, participate in activities, or exercise [13]. For health promotion programs to be effective, health care providers need to pay attention to participants' ATA. Identifying the factors associated with ATA is an important first step prior to the exploration of possible interventions.

Literature has reported inconsistent results on the association of demographic factors (age, sex, education, marriage and living status, income) with ATA [12-15]. Negative ATA is often associated with many chronic diseases such as hypertension, diabetes and coronary heart disease [11,16], respiratory disease [17], arthritis, asthma, and depression [13,18]. In addition, participating in leisure activities [19], regular exercise [20,21], and volunteer service [22] has been shown to reduce the relationship between ATA and health. Most of the past studies were conducted in western countries, and this issue has been rarely investigated in an Asian population. Since ATA may be affected by living environment and societal culture, the associated factors of ATA in an Asian population needs to be explored. This issue is urgent to be answered especially as Taiwan experiences the fastest rate of aging in the world; the proportion of people over 65 was $8.6 \%$ in 2000 and is expected to rise to $20 \%$ by $2027[23,24]$.

The purposes of this study were (1) to identify specific demographic and health-related factors associated with ATA, and (2) to identify the specific activities that are significantly associated with ATA over and above the demographic and health-related factors in a group of middle-aged and older adults from analysis of population-representative national data.

\section{Materials and Methods}

The Taiwan Longitudinal Study on Aging (TLSA) is a population-based longitudinal study of a nationally representative random sample of adults aged 58 years and older that begun in 1989 and followed up every 3 to 4 years. The details and design of the TLSA have been described elsewhere $[25,26]$.

Data on participants collected in 2011 (TLSA 2011) were used in this cross-sectional study since this was the first year the TAAQ-SF questionnaire was used in this survey to measure ATA. We selected responses without missing data on the Taiwanese Attitude toward Aging Questionnaire Short Form (TAAQ-SF) [27]. To ensure the validity of responses on the TAAQ-SF from the study participants, we further selected those who were cognitively intact, as indicated by a score of $\geq 6$ on the Short Portable Mental Status Questionnaire (SPMSQ) [28]. The current study was approved by the Institutional Review Board of the Health Promotion Administration of the Ministry of Health and Welfare (Approval No. 5 BHP-2007-002). Before recruitment, all participants received a proper explanation of the study and provided informed consent for inclusion in the study. Participants who could read and write signed the written consent documents; those who could not read or write impressed their name stamps or fingerprints with the assistance of family members. The TLSA 2011 variables used in this study were as follows.

\subsection{Demographic Variables}

The participants' demographic characteristics consisted of age, gender, education ( $\leq 6$ years, $7-12$ years, and $\geq 13$ years), marital/cohabitation status (living with or without spouse/partner), and financial satisfaction (satisfied, average, and dissatisfied).

\subsection{Attitude toward Aging (ATA)}

Participants' ATA were indicated by the total score of the TAAQ-SF that consists of eleven items: (1) you feel that you are old, (2) you feel you have time to pursue your own interests, (3) you feel it is a privilege to grow old, (4) you feel that you cannot take care of yourself, (5) you feel that you could help your family, (6) you feel that you are frail, (7) you feel that you are pleasant and joyful, (8) you feel your mind is not clear, (9) you feel you are kind and warm, (10) you feel unsafe, and (11) you feel satisfied with your current life. Each item was rated on a 5-point Likert scale from 1 (strongly disagree) to 5 (strongly agree). 
Before summing up the total score, items one, four, six, eight, and ten were reverse-scored to make all the items measure a positive ATA. The total score ranged from 11 to 55, with a higher score indicating more positive ATA. The psychometric properties of TAAQ-SF was examined and confirmed in a previous study [27].

\subsection{Health-Related Variables}

\subsubsection{Depression}

Participants' depressive symptoms were assessed with the 10-item short form of the Center for Epidemiological Studies Depression Scale (CESD-10) [29]. The total score range is from 0 to 30, with a higher score indicating greater severity of depressive symptoms. Participants were assigned to either the depressed group (CESD-10 $\geq 10$ ) or the nondepressed group (CESD-10 $\leq$ ) [30].

\subsubsection{Physical Function}

Participants' physical function was assessed with three scales: mobility (lift/carry $12 \mathrm{~kg}$, walk 200 to $300 \mathrm{~m}$, and climb stairs to second or third floor), instrumental activities of daily living (IADL) (shopping for personal items, ability to handle finances, mode of transportation, heavy housework, housekeeping, ability to use telephone, food preparation, responsibility for own medications, and laundry), and activities of daily living (ADL) (bathing, dressing, feeding, transfer, mobility, and toilet use). An individual was considered "independent" if the participant answered, "without difficulty" on all items on the scale; otherwise, as "dependent". Physical function was further categorized into four groups: independent on all 3 scales, dependent on any 1 scale, dependent on any 2 scales, and dependent on all 3 scales.

\subsubsection{Comorbidity}

Total numbers of co-morbidities were summed from the following 15 comorbidities: hypertension, diabetes, cardiovascular disease, stroke (or transient ischemic attack), cancer/malignant tumor, chronic obstructive pulmonary disease (COPD), asthma, arthritis, hepatobiliary disease, hip fracture, cataract, glaucoma, chronic kidney disease, gout, and hyperlipidemia.

\subsection{Activities}

\subsubsection{Physical Exercise}

Participants were grouped based on exercise frequency (no exercise, $<3$ or $\geq 3$ times per week), exercise time ( $<30$ or $\geq 30$ min per session), and exercise intensity (do not sweat, sweat a little, or sweat a lot). Those who did not exercise were in the "no exercise" group. Those who exercised $\geq 3$ times per week, $\geq 30$ min each time, and sweated a little or a lot during exercise were in the "regular exercise" group, whereas all others were in the "irregular exercise" group.

\subsubsection{Social Service (Volunteer) Work and Leisure Activities}

Participants were asked if they participated in social service (volunteer) work, and any of the following leisure activities: listening to music/radio, reading newspapers/magazines/ books, going online (not interacting with other persons), going online (interacting with other persons), chatting with relatives/friends, playing chess or cards, gardening (horticulture), taking a walk, bicycling, engaging in outdoor fitness activities (such as jogging, playing ball games, hiking, mountain climbing, etc.), and group activities (such as singing, dancing, Tai Chi, Wai-Tan-Kung, etc.).

\subsection{Data Analysis}

Data were analyzed in SPSS 17.0 (2008 SPSS, Inc., Chicago, IL, USA). Participants' characteristics are reported as descriptive statistics. The associations between the dependent variable (ATA) and categorical independent variables (demographic variables and 
health-related variables) were examined with one-way analysis of variance (ANOVA). If global ANOVA showed significant results, a post hoc analysis was performed. The effect size (Eta squared, $\eta^{2}$ ) was reported for those who showed significant association. The $\eta^{2}$ was calculated as $\frac{\text { sum of squares between groups }}{\text { total sum of squares }}$, where 0.01 was small, 0.06 was medium, and 0.14 was a large effect [31]. The association of ATA with the continuous independent variable (total number of co-morbidities) was examined with Spearman's correlation coefficient $\left(r_{s}\right)$. A correlation coefficient of $0.10-0.29$ was considered small; $0.30-0.49$, medium, and 0.50-1.0, large [31].

To identify the demographic and health-related variables that were significantly associated with ATA, those which showed significant associations with the dependent variable (ATA) in the bivariate analysis were entered into the multiple regression analysis. To identify the various activities associated with ATA, a hierarchical multiple regression analysis was performed for each activity category, with demographic and health-related variables controlled for. The standardized Beta $(\beta), \mathrm{t}$ statistics, and $\mathrm{p}$ value were reported for comparison of the contributions among the factors.

\section{Results}

\subsection{Sample Characteristics}

The 2011 TLSA had 3727 respondents aged 58 to 101 years who completed the interview. In this study, we selected those without missing data on any of the TAAQ-SF items $(n=3272)$ and without impaired cognition (SPMSQ $\geq 6)(n=3054)$. Thus, a final total of 3054 respondents' data was used for analysis in this study.

The characteristics of our participants are reported in Table 1 . The participants had a mean age of $69.1(\mathrm{SD}=9.0)$. Most were men $(50.5 \%)$ with $\leq 6$ years of education $(62.6 \%)$ who were living with a spouse/partner (71.9\%) and were satisfied with their financial condition (43.4\%). On average, our participants had 2.2 co-morbidities, $39.1 \%$ were dependent on at least one physical function scale, and $15.3 \%$ were depressed. Just under one third $(31.5 \%)$ did not exercise at all, and $21.3 \%$ participated in volunteer service. The top five leisure activities were chatting with friends/relatives (73.4\%), taking a walk (67.1\%), reading newspapers/magazines $(47.9 \%)$, listening to music/radio $(45.1 \%)$, and gardening (horticulture) (41.9\%).

Table 1. Participant characteristics $(n=3054)$.

\begin{tabular}{|c|c|c|}
\hline Social Demographic Characteristics & Mean \pm SD & n $(\%)$ \\
\hline Age (years) & $69.1 \pm 9.0$ & \\
\hline \multicolumn{3}{|l|}{ Gender } \\
\hline Men & & $1543(50.5 \%)$ \\
\hline Women & & $1511(49.5 \%)$ \\
\hline \multicolumn{3}{|l|}{ Education } \\
\hline$\leq 6$ years & & $1912(62.6 \%)$ \\
\hline $7-12$ years & & $803(26.3 \%)$ \\
\hline$\geq 13$ years & & $339(11.1 \%)$ \\
\hline \multicolumn{3}{|l|}{ Marital/cohabitation status, $(n=3008)$} \\
\hline Without spouse/partner & & $844(28.1 \%)$ \\
\hline With spouse/partner & & $2164(71.9 \%)$ \\
\hline \multicolumn{3}{|l|}{ Financial satisfaction } \\
\hline Dissatisfied & & $564(18.5 \%)$ \\
\hline Acceptable & & $1165(38.2 \%)$ \\
\hline Satisfied & & $1324(43.4 \%)$ \\
\hline Attitude toward aging (TAAQ-SF) (scores) & $40.6 \pm 6.0$ & \\
\hline \multicolumn{3}{|l|}{ Health-related variables } \\
\hline Depression $(C E S D-10)(n=3052)$ & & \\
\hline Depressed (CESD-10 $\geq 10)$ & & $467(15.3 \%)$ \\
\hline
\end{tabular}


Table 1. Cont.

\begin{tabular}{|c|c|c|}
\hline Social Demographic Characteristics & Mean \pm SD & n (\%) \\
\hline \multicolumn{3}{|l|}{ Physical function ( $\mathrm{n}=3045$ ) } \\
\hline All 3 scales dependent & & $190(6.2 \%)$ \\
\hline Any 2 scales dependent & & $526(17.3 \%)$ \\
\hline Any 1 scale dependent & & $474(15.6 \%)$ \\
\hline All 3 scales independent & & $1855(60.9 \%)$ \\
\hline Numbers of co-morbidities & $2.2 \pm 1.8$ & \\
\hline \multicolumn{3}{|l|}{ Activities } \\
\hline \multicolumn{3}{|l|}{ Physical exercise $(n=2996)$} \\
\hline No exercise & & $944(31.5 \%)$ \\
\hline Irregular exercise & & $989(33.0 \%)$ \\
\hline Regular exercise & & $1063(35.5 \%)$ \\
\hline Volunteer service (yes) & & $454(14.9 \%)$ \\
\hline \multicolumn{3}{|l|}{ Leisure activity } \\
\hline Listening to music/radio & & $1378(45.1 \%)$ \\
\hline Reading newspaper/magazines & & $1464(47.9 \%)$ \\
\hline Going online (no interaction with other persons) & & $358(11.7 \%)$ \\
\hline Going online (interaction with other persons) & & $171(5.6 \%)$ \\
\hline Playing chess or cards & & $331(10.8 \%)$ \\
\hline Chatting with relatives/friends & & $2242(73.4 \%)$ \\
\hline Gardening (horticulture) & & $1279(41.9 \%)$ \\
\hline Taking a walk & & $2048(67.1 \%)$ \\
\hline Biking & & $816(26.7 \%)$ \\
\hline Outdoor fitness & & $572(18.7 \%)$ \\
\hline Group activity & & $482(15.8 \%)$ \\
\hline
\end{tabular}

Abbreviations: CESD-10, the 10-item short form of the Center for Epidemiological Studies Depression Scale; TAAQ-SF, Taiwanese Attitude toward Aging Questionnaire Short Form.

\subsection{Results of Bivariate Analysis}

The dependent variable was ATA (TAAQ-SF), and the independent variables were the demographic and health-related factors. The results of ANOVA indicated that all independent variables were significantly associated with ATA (Table 2). Depression $\left(\eta^{2}=0.27\right)$ and physical function $\left(\eta^{2}=0.26\right)$ showed large effect sizes. Financial satisfaction $\left(\eta^{2}=0.12\right)$, age $\left(\eta^{2}=0.10\right)$, and education $\left(\eta^{2}=0.06\right)$ showed medium effect sizes. The total number of co-morbidities showed a medium relationship with ATA $\left(\mathrm{r}_{\mathrm{s}}=-0.315, p<0.001\right)$.

Table 2. Results of bivariate analysis: the association between attitude toward aging (ATA), and each demographic and health-related factor.

\begin{tabular}{|c|c|c|c|c|c|}
\hline & $\mathbf{n}$ & Mean (SD) of ATA & $p$ & Post Hoc & Eta Squared \\
\hline \multicolumn{6}{|c|}{ Demographic Factors } \\
\hline \multicolumn{6}{|c|}{ Age } \\
\hline $58-64$ & 1254 & $42.6(5.4)$ & \multirow{4}{*}{0.000} & & \multirow{4}{*}{0.10} \\
\hline $65-74$ & 954 & $40.3(5.8)$ & & All pairwise & \\
\hline $75-84$ & 645 & $38.4(6.2)$ & & comparisons $(p<0.05)$ & \\
\hline $85-98$ & 201 & $37.1(5.3)$ & & & \\
\hline \multicolumn{6}{|c|}{ Gender } \\
\hline Men & 1543 & $41.2(5.9)$ & \multirow{3}{*}{0.000} & & \multirow{2}{*}{0.009} \\
\hline Women & 1511 & $40.0(6.0)$ & & & \\
\hline \multicolumn{5}{|c|}{ Education } & \\
\hline$\leq 6$ years & 1912 & $39.6(6.1)$ & \multirow{3}{*}{0.000} & & \multirow{3}{*}{0.06} \\
\hline $7-12$ years & 803 & $41.9(5.5)$ & & All pairwise & \\
\hline$\geq 13$ years & 339 & $43.5(5.1)$ & & & \\
\hline
\end{tabular}


Table 2. Cont.

\begin{tabular}{|c|c|c|c|c|c|}
\hline & $\mathbf{n}$ & Mean (SD) of ATA & $p$ & Post Hoc & Eta Squared \\
\hline \multicolumn{6}{|c|}{ Marital/cohabitation status } \\
\hline Without spouse/partner & 844 & $38.5(6.2)$ & \multirow{2}{*}{0.000} & & \multirow[b]{2}{*}{0.05} \\
\hline With spouse/partner & 2164 & $41.5(5.7)$ & & & \\
\hline \multicolumn{6}{|c|}{ Financial satisfaction } \\
\hline Dissatisfied & 564 & $36.9(6.7)$ & \multirow{3}{*}{0.000} & & \multirow{3}{*}{0.12} \\
\hline Acceptable & 1165 & $40.3(5.4)$ & & All pairwise & \\
\hline Satisfied & 1324 & $42.5(5.3)$ & & & \\
\hline \multicolumn{6}{|c|}{$\begin{array}{l}\text { Health-related Factors } \\
\text { Depression (CESD-10) }\end{array}$} \\
\hline Depressed (CESD-10 $\geq 10)$ & 467 & $33.3(5.8)$ & \multirow{3}{*}{0.000} & & \multirow{2}{*}{0.27} \\
\hline Not depressed (CESD-10 $\leq 9)$ & 2585 & $42.0(5.0)$ & & & \\
\hline \multicolumn{5}{|c|}{ Physical function } & \\
\hline All 3 scales dependent & 190 & $32.8(6.0)$ & \multirow{4}{*}{0.000} & & \multirow{4}{*}{0.26} \\
\hline Any 2 scales dependent & 526 & $36.7(5.8)$ & & All pairwise & \\
\hline Any 1 scale dependent & 474 & $39.8(5.2)$ & & comparisons $(p<0.05)$ & \\
\hline All 3 scales independent & 1855 & $42.8(4.8)$ & & & \\
\hline
\end{tabular}

Abbreviations: CESD-10, the 10-item short form of the Center for Epidemiological Studies Depression Scale.

\subsection{Results of Multiple Regression Analysis: Factors Associated with ATA}

All of the independent variables, except gender, were significantly associated with ATA (Table 3). The total variance explained by the model was $46.7 \%$ (adjusted $\mathrm{R}^{2}=46.4 \%$, $\left.\mathrm{F}_{(14,2973)}=185.9036, p<0.001\right)$. In the model, depression $(\beta=-0.32, p<0.001)$, financial satisfaction $(\beta=0.28, p<0.001)$, and dependent on all 3 physical function scales $(\beta=-0.23$, $p<0.001)$ showed the largest contributions to the model.

Table 3. Results of multiple regression analysis: factors significantly associated with attitude toward aging.

\begin{tabular}{|c|c|c|}
\hline & $\beta$ & $\mathbf{t}$ \\
\hline \multicolumn{3}{|l|}{ Age (Reference: $58-64)$} \\
\hline $65-74$ & -0.06 & $-4.04^{* * *}$ \\
\hline $75-84$ & -0.10 & $-5.79 * * *$ \\
\hline $85-98$ & -0.10 & $-6.05^{* * *}$ \\
\hline \multicolumn{3}{|l|}{ Gender (Reference: women) } \\
\hline Men & 0.02 & -1.14 \\
\hline \multicolumn{3}{|l|}{ Education (Reference: $\leq 6$ years) } \\
\hline Education ( $7-12$ years) & 0.07 & $4.71^{* * *}$ \\
\hline Education ( $>12$ years) & 0.07 & $5.04 * * *$ \\
\hline \multicolumn{3}{|c|}{$\begin{array}{c}\text { Marital/cohabitation status (Reference: without } \\
\text { spouse/partner) }\end{array}$} \\
\hline With spouse/partner & 0.04 & $2.97^{* *}$ \\
\hline \multicolumn{3}{|c|}{ Financial status (Reference: dissatisfied) } \\
\hline Average & 0.13 & $6.81^{* * *}$ \\
\hline Satisfied & 0.28 & $14.21^{* * *}$ \\
\hline Number of co-morbidities & -0.09 & $-5.97 * * *$ \\
\hline \multicolumn{3}{|c|}{ Depression (Reference: not depressed, CESD-10 $\leq 9$ ) } \\
\hline Depressed (CESD-10 $\geq 10)$ & -0.32 & $-20.82 * * *$ \\
\hline \multicolumn{3}{|c|}{ Physical function (Reference: independent in all 3) } \\
\hline Any 1 scale dependent & -0.08 & $-5.69^{* * *}$ \\
\hline Any 2 scales dependent & -0.18 & $-10.68^{* * *}$ \\
\hline All 3 scales dependent & -0.23 & $-14.46^{* * *}$ \\
\hline
\end{tabular}

Abbreviations: CESD-10, the 10-item short form of the Center for Epidemiological Studies Depression Scale; $\beta$, standardized coefficient. ${ }^{* *} p<0.01 ;{ }^{* * *} p<0.001$. 


\subsection{Activities Associated with ATA}

Both physical exercise and volunteering, each individually, were found significant to ATA controlled for the demographic and health-related factors (Table 4). Both regular and irregular exercise, compared with no exercise, showed significant beneficial effects on ATA. Regular exercise $(\beta=0.10, p<0.001)$ showed a larger effect on ATA than did irregular exercise $(\beta=0.05, p<0.01)$ and volunteer service $(\beta=0.07, p<0.001)$. As to the leisure activities, the following six activities showed significant influences on the ATA: gardening $(\beta=0.08, p<0.001)$, outdoor fitness activities $(\beta=0.07, p<0.001)$, group activities $(\beta=0.06, p<0.001)$, reading newspapers $/$ magazines $/$ books $(\beta=0.05, p=0.01)$, chatting with friends/relatives $(\beta=0.04, p<0.01)$, and listening to radio/music $(\beta=0.03, p<0.05)$.

Table 4. The association of physical exercise, volunteer activities, and leisure activities with attitude toward aging controlling for significant demographic and health-related factors.

\begin{tabular}{|c|c|c|}
\hline Variables & $\beta$ & $\mathrm{t}$ \\
\hline \multicolumn{3}{|l|}{ Physical exercise (Reference: no exercise) † } \\
\hline Irregular & 0.05 & $3.27^{* *}$ \\
\hline Regular & 0.10 & $6.15^{* * *}$ \\
\hline \multicolumn{3}{|l|}{ Volunteer activities (Reference: no participation) † } \\
\hline Volunteer service (participation) & 0.07 & $5.23^{* * *}$ \\
\hline \multicolumn{3}{|l|}{ Leisure activities $\dagger$} \\
\hline Listening to radio/music & 0.03 & $2.26^{*}$ \\
\hline Reading newspapers/magazines/books & 0.05 & $3.40 * *$ \\
\hline Going online (no interaction with other person) & 0.01 & 0.40 \\
\hline Going online (interaction with other persons) & 0.01 & 0.89 \\
\hline Playing chess or cards & 0.02 & 1.75 \\
\hline Chatting with friends/relatives & 0.04 & $2.85^{* *}$ \\
\hline Gardening (horticulture) & 0.08 & $5.43^{* * *}$ \\
\hline Taking a walk & 0.03 & 1.82 \\
\hline Bicycling & 0.02 & 1.71 \\
\hline Outdoor fitness & 0.07 & $4.63^{* * *}$ \\
\hline Group activity & 0.06 & $3.96^{* * *}$ \\
\hline
\end{tabular}

†: model adjusted demographic factors (age, education, marital and cohabitation status, and financial satisfaction) and health-related factors (numbers of co-morbidities, depression, and physical function). Abbreviation: $\beta$, standardized regression coefficient. ${ }^{*} p<0.05,{ }^{* *} p<0.01,{ }^{* * *} p<0.001$.

\section{Discussion}

The purposes of this study were to identify the factors associated with ATA and the activities beneficial to ATA after the identified associated factors being controlled for in the middle-aged and older Taiwanese population. The results of this study revealed that higher education, living with others, and financial satisfaction are protective factors to ATA, whereas advanced age, higher numbers of co-morbidities, depressive mood, and physical functioning dependency were risk factors to ATA. Beyond the influence of demographic and health-related factors, participation of physical exercise, volunteer service, and six leisure activities were all beneficial to ATA in the middle-aged to older adults.

Among the identified factors associated with ATA, the only non-modifiable factor was advanced age. Consistent with previous studies, older adults' views on aging often reflect societal beliefs and stereotypes about aging, which primarily convey the negative aspects of growing old [15,32]. Higher education, living with others, satisfaction with financial status, absence of depression, independent physical functions (in mobility, IADL, and AD), and fewer co-morbidities were modifiable beneficial factors to ATA and are worthy of consideration for government policy makers in the development of strategies for enhancing ATA for future middle-aged to older generations. Consistent with the literature, higher education was associated with positive ATA $[13,14]$. Specifically, people with higher education experienced significantly less psychosocial loss associated with increasing age than those with lower education, measured by Attitude toward Aging Questionnaire (AAQ) [13]. Living alone and unsatisfactory financial condition were associated with negative ATA [12]. In 
terms of marriage status, those who never married showed significantly less psychological growth as measured by AAQ whereas those who were separated/divorced and widowed also showed less, but not significant, on psychological growth (positive aspect of wisdom and generativity that adults can feel as they grow older) [13].

The influence of gender on ATA was found to be inconsistent from previous studies, in which some reported no influence [12-14] whereas others found men showed more positive ATA than women [15]. Our results found the influence of gender on ATA was confounded by education. Gender was significantly associated with ATA in bivariate analysis, in which men showed significantly more positive attitudes than women. This finding was consistent with a previous study [15]. However, gender was not a significant factor with ATA in the multiple regression analysis because it was also significantly associated with education. Thus, when education was entered into the model, gender did not make a significant contribution to it.

Physical exercise and volunteer service were found to be beneficial to ATA over and above the demographic and health-related factors. Compared to the no exercise group, both regular and irregular physical exercise groups showed significant positive influence on ATA, with the regular exercise group showing greater influence than the irregular exercise group. The benefit of participating in volunteer service to ATA might be through the following mechanisms: more opportunities for social interactions, increased sense of self-worth, greater sense of accomplishment, and better self-rated health [22,33].

Among the leisure activities investigated, six activities showed the significant influential effects on ATA. To the best of our understanding, this is the first study to explore the specific activities which have significant impact on the ATA over and above the other influential factors. Our results revealed that gardening was the most influential activity to ATA. Gardening has been reported to improve survival rates in those with mobility limitations or depressed people aged $\geq 50$ years [33]. Both the outdoor fitness and group activities were considered physical leisure activities. These activities are known to be effective in improving physical function (cardiopulmonary, musculoskeletal), mental health, and self-rated health [34,35]. Reading newspapers/magazines/books and listening to radio/music were considered as cognitive leisure activities [36]. Cognitive leisure activities provide intellectual stimulation and thus are beneficial to cognitive function, maintaining motivation, and relieving psychological stress [36]. Chatting with friends/relatives provides important social support.

To the best of our knowledge, this is the first study to examine the factors associated with ATA and to explore the activities associated with ATA while controlling for demographic and health-related factors in a Taiwanese population. The strength of this study is that the results are based on a nationally representative dataset. However, readers need to be aware of the following when generalizing the results. First, these results were based on a nationally representative Taiwanese population and we included those who were cognitively intact (SPMSQ $\geq 6$ ). These results can be generalized only to similar populations. Second, the causal relationships of the identified factors that are beneficial and detrimental to ATA could not be ascertained in this cross-sectional study. Future studies are recommended to further examining the effects of these identified factors on ATA and health, especially the effects of interventions featuring participation in the identified activities on negative ATA. Further investigations focused on different subgroups by gender, financial status, living alone or not, marital status, and living in larger/smaller cities are also recommended.

\section{Conclusions}

Advanced age, a higher number of co-morbidities, living alone, depression, and dependence on physical function were detrimental to ATA. Higher education, financial satisfaction, and participation in physical exercise, volunteer service, and six leisure activities were all beneficial to ATA. The identified beneficial and detrimental factors provide valuable information for future studies and for developing health promotion interventions. 


\begin{abstract}
Author Contributions: Conceptualization, S.-H.L., C.-Y.Y. and C.-Y.W.; methodology, S.-H.L., C.-J.Y. and C.-Y.W.; writing-original draft preparation, C.-Y.Y. and C.-Y.W.; writing-review and editing, S.-H.L., C.-J.Y., C.-Y.Y., C.-Y.W. and M.-C.L.; supervision, M.-C.L.; project administration, M.-C.L.; funding acquisition, M.-C.L. All authors have read and agreed to the published version of the manuscript.
\end{abstract}

Funding: This work was supported by Ministry of Health and Welfare, Taiwan (grant number: 25 M06M2346 awarded to M.-C.L.).

Institutional Review Board Statement: The study was conducted according to the guidelines of the Declaration of Helsinki, and approved by the Institutional Review Board of the Health Promotion Administration of the Ministry of Health and Welfare (Approval No. 5 BHP-2007-002).

Informed Consent Statement: Not applicable.

Data Availability Statement: TLSA data is a national study project, and its data is open to those who have been approved to use it for analysis (http:/ / nhis.nhri.org.tw/ accessed on 13 October 2017).

Conflicts of Interest: The authors declare no conflict of interest.

\title{
References
}

1. Levy, B. Stereotype embodiment. A psychosocial approach to aging. Curr. Dir. Psychol. Sci. 2009, 18, 332-336. [CrossRef]

2. Lawton, M.P. The Philadelphia geriatric center morale scale: A revision. J. Gerontol. 1975, 30, 85-89. [CrossRef] [PubMed]

3. Laidlaw, K.; Power, M.J.; Schmidt, S.; The WHOQOL-OLD Group. The attitudes to ageing questionnaire (AAQ): Development and psychometric properties. Int. J. Geriatr. Psychiatry 2007, 22, 367-379. [CrossRef] [PubMed]

4. Sarkisian, C.A.; Steers, W.N.; Hays, R.D.; Mangione, C.M. Development of the 12-item Expectations Regarding Aging Survey. Gerontologist 2005, 45, 240-248. [CrossRef]

5. Andrews, R.M.; Tan, E.J.; Varma, V.R.; Rebok, G.W.; Romani, W.A.; Seeman, T.E.; Gruenewald, T.L.; Tanner, E.K.; Carlson, M.C. Positive Aging Expectations Are Associated With Physical Activity Among Urban-Dwelling Older Adults. Gerontologist 2017, 57 (Suppl. S2), S178-S186. [CrossRef] [PubMed]

6. Levy, B.R.; Slade, M.D.; Kunkel, S.R.; Kasl, S.V. Longevity increased by positive self-perceptions of aging. J. Pers. Soc. Psychol. 2002, 83, 261-270. [CrossRef] [PubMed]

7. Levy, B.R.; Meyers, L.M. Preventive health behaviors influenced by self-perceptions of aging. Prev. Med. 2004, 39, 625-629. [CrossRef]

8. Gale, C.; Cooper, C. Attitudes to ageing and change in frailty status: The English longitudinal study on ageing. Gerontology 2018, 64, 58-66. [CrossRef]

9. Wurm, S.; Tomasik, M.J.; Tesch-Romer, C. Serious health events and their impact on changes in subjective health and life satisfaction: The role of age and appositive view on ageing. Eur. J. Ageing 2008, 5, 117-127. [CrossRef]

10. Siebert, J.S.; Wahl, H.W.; Degen, C.; Schroder, J. Attitude toward aging as a risk factor for cognitive disorder in old age: 12-year evidence from the ILSE study. Psychol. Aging 2018, 33, 461-472. [CrossRef]

11. Levy, B.R.; Zonderman, A.B.; Slade, M.D.; Ferrucci, L. Negative age stereoptypes held earlier in life predict cardiovascular events in later life. Psychol. Sci. 2009, 20, 296-298. [CrossRef] [PubMed]

12. Moser, C.; Spagnoli, J.; Santos-Eggimann, B. Self-perception of aging and vulnerability to adverse outcomes at the age of 65-70 years. J. Gerontol. B Psychol. Sci. Soc. Sci. 2011, 66, 675-680. [CrossRef]

13. Thorpe, A.M.; Pearson, J.F.; Schluter, P.J.; Spittlehouse, J.K.; Joyce, P.R. Attitudes to aging in midlife are related to health conditions and mood. Int. Psychoger. 2014, 26, 2061-2071. [CrossRef] [PubMed]

14. Zielinska-Wieczkowska, H.; Sas, K. The Sense of Coherence, Self-Perception of Aging and the Occurrence of Depression Among the Participants of the University of the Third Age Depending on Socio-Demographic Factors. Clin. Inter. Aging 2020, 15, 1481-1491. [CrossRef] [PubMed]

15. Janeckova, H.; Dragomirecka, E.; Holmerova, I.; Vankova, H. The attitudes of older adults living in institutions and their caregivers to ageing. Cent. Eur. J. Public Health 2013, 21, 63-71. [CrossRef]

16. Demakakos, P.; Gjonca, E.; Nazroo, J. Age Identity, Age Perceptions, and Health: Evidence from the English Longitudinal Study of Ageing. Ann. N. Y. Acad. Sci. 2007, 1114, 279-287. [CrossRef]

17. Levy, B.R.; Myers, L.M. Relationship between respiratory mortality and self-perceptions of aging. Psychol. Health 2005, 20, 553-564. [CrossRef]

18. Laidlaw, K.; Kishita, N.; Shenkin, S.D.; Power, M.J. Development of a short form of the Attitudes to Ageing Questionnaire (AAQ). Int. J. Geriatr. Psychiatry 2018, 33, 113-121. [CrossRef]

19. Hicks, S.A.; Siedlecki, K.L. Leisure activity engagement and positive affect partially mediate the relationship between positive views on aging and physical health. J. Gerontol. B Psychol. Sci. Soc. Sci. 2017, 72, 259-267. [CrossRef]

20. Elsawy, B.; Higgins, K.E. Physical activity guidelines for older adults. Am. Fam. Physician 2010, 81, 55-59. 
21. Kim, S.H. Older people's expectations regarding ageing, health-promoting behavior and health status. J. Adv. Nurs. 2009, 65, 84-91. [CrossRef] [PubMed]

22. Liu, Y.; Duan, Y.; Xu, L. Volunteer service and positive attitudes toward aging among Chinese older adults: The mediating role of health. Soc. Sci. Med. 2020, 265, 113535. [CrossRef] [PubMed]

23. Department of Health EY. Taiwan Public Health Report 2017; Ministry of Health and Welfare: Taipei, Taiwan, 2018.

24. National Development Council. Population Projections for Taiwan: 2018-2065; Department of Human Resources Development, National Development Concil: Taipei, Taiwan, 2018.

25. Liang, J.; Wang, C.N.; Xu, X.; Hsu, H.C.; Lin, H.S.; Lin, Y.H. Trajectory of functional status among older Taiwanese: Gender and age variations. Soc. Sci. Med. 2010, 71, 1208-1217. [CrossRef] [PubMed]

26. Tai, C.J.; Chen, J.H.; Tseng, T.G.; Lin, Y.T.; Hsiao, Y.H.; Lee, M.C.; Yang, Y.H. Prediction of railty and dementia using oral health impact profle from a population-based survey. Int. J. Environ. Res. Public Health 2020, 17, 1997. [CrossRef]

27. Yeh, C.J.; Yang, C.Y.; Lee, S.H.; Wang, C.Y.; Lee, M.C. Psychometric Properties of a Taiwanese Attitude toward Aging QuestionnaireShort Form Used in the Taiwan Longitudinal Study on Aging. Int. J. Gerontol. 2022, in press.

28. Yen, C.-H.; Yeh, C.-J.; Wang, C.-C.; Liao, W.-C.; Chen, S.-C.; Chen, C.-C.; Liang, J.; Lai, T.-J.; Lin, H.-S.; Lee, S.-H.; et al. Determinants of cognitive impairment over time among the elderly in Taiwan: Results of the national longitudinal study. Arch. Gerontol. Geriatr. 2010, 50 (Suppl. S1), 553-557. [CrossRef]

29. Lee, K.L.; Ou, Y.L.; Chen, S.H.; Weng, L.J. The psychometric properties of a short form of the CES-D used in the Taiwan longitudinal study on aging. Formosa J. Mental. Health 2009, 22, 383-410.

30. Lee, C.T.; Yeh, C.J.; Lee, M.C.; Lin, H.S.; Chen, C.H.; Hsieh, M.H.; Yen, C.H.; Lai, T.J. Social support and mobility limitation as modifiable predictors of improvement in depressive symptoms in the elderly: Results of a national longitudinal study. Arch. Gerontol. Geriatr. 2012, 55, 530-538. [CrossRef]

31. Pallant, J. SPSS Survival Manual: A Step by Step Guide to Data Analysis Using IBM SPSS, 6th ed.; McGraw-Hill House: Maidenhead Berkshire, UK, 2016.

32. Diehl, M.; Wahl, H.-W.; Barrett, A.E.; Brothers, A.F.; Miche, M.; Montepare, J.M.; Westerhof, G.J.; Wurm, S. Awareness of aging: Theoretical considerations on an emerging concept. Dev. Rev. 2014, 34, 93-113. [CrossRef]

33. Leng, C.H.; Wang, J.D. Daily home gardening improved survival for older people with mobility limitations: An 11-year follow-up study in Taiwan. Clin. Inv. Aging 2016, 11, 947-959.

34. Wurm, S.; Thomasik, M.J.; Tesch-Romer, C. On the importance of a positive view on aging for physical exercise among middleaged and older adults: Cross-sectional and longitudinal findings. Psychol. Health 2010, 25, 25-42. [CrossRef] [PubMed]

35. Beyer, A.K.; Wolff, J.K.; Warner, L.M.; Schüz, B.; Wurm, S. The role of physical activity in the relationship between self-perceptions of ageing and self-rated health in older adults. Psychol. Health 2015, 30, 671-685. [CrossRef] [PubMed]

36. Iizuka, A.; Suzuki, H.; Ogawa, S.; Kobayashi-Cuya, K.E.; Kobayashi, M.; Takebayashi, T.; Fujiwara, Y. Can cognitive leisure activity prevent cognitive decline in older adults? A systematic review of intervention studies. Geriatr. Gerontol. Int. 2019, 19, 469-482. [CrossRef] [PubMed] 